\title{
SOME PROPERTIES OF CERTAIN EXTREMAL DECOMPOSITIONS OF A RECTANGLE
}

\section{FRIEDRICH HUCKEMANN}

1. General notation. For two distinct points $a, b$ of the complex plane $C$ or of the two-dimensional Euclidean space $\boldsymbol{R}^{2},[a, b]$ is the closed straight line segment joining these two points; we put $\langle a, b]=$ $[a, b]-\{a\},[a, b\rangle=[a, b]-\{b\},\langle a, b\rangle=[a, b]-\{a, b\},[a, a]=$ $\{a\}$; for a subset $S$ of $C$ or of $\boldsymbol{R}^{2}, \bar{S}$ is the closure and $\partial S$ is the boundary of $S$.

For fixed $M>0, Q$ denotes the rectangle $\langle 0, M\rangle \times\langle 0, i\rangle$. In the course of this investigation, 4 or 5 or 6 boundary points of $Q$ will be distinguished, and $Q$ will be considered as quadrilateral or pentagon or hexagon accordingly. Choosing $x \in\langle 0, M\rangle$ and singling out the distinct points $0, x, M, M+i, i$ on $\partial Q, Q$ becomes a pentagon with sides $[0, x],[x, M],[M, M+i],[M+i, i],[i, 0]$. Selecting two nonadjacent sides $s_{1}$ and $s_{2}$ out of these five sides, the remaining sides $s_{3}, s_{4}, s_{5}$ are so numbered that $s_{3}$ and $s_{4}$ have a common point, denoted by $P$. We denote by $\Re=\Re\left(s_{1}, s_{2} ; P\right)$ the class of continua $K$ in $\bar{Q}$ with the properties

(i) $K$ connects $P$ and $s_{5}$,

(ii) $K$ is disjoint from $s_{1} \cup s_{2}$.

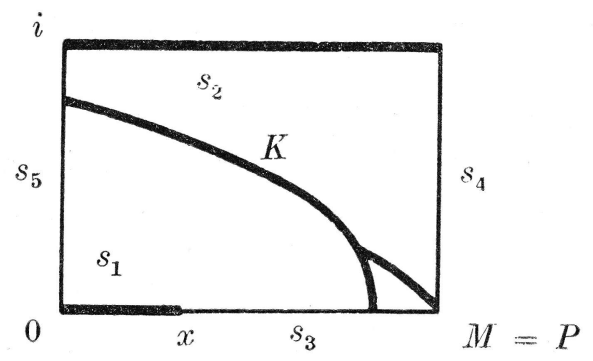

Figure 1 
$K$ separates $s_{1}$ from $s_{2}$ in $\bar{Q}$ and decomposes $Q$ into several simply connected components. The component of $(\bar{Q}-K) \cap Q$ which has $s_{j}$ on its boundary $(j=1,2)$ we call $D_{j}=D_{j}\left(s_{1}, s_{2} ; K\right) . K_{j}=K \cap \partial D_{j}$ is connected; $M_{j}=M_{j}\left(s_{1}, s_{2} ; K\right)$ denotes the extremal distance between $s_{j}$ and $K_{j}$ in $D_{j} ; M_{j}$ is then the modulus of the quadrilateral $D_{j}$ with respect to the pair $\left(s_{j}, K_{j}\right)$ of sides. For a domain $D \subset C$ and two connected compact sets $\gamma_{1}, \gamma_{2} \subset \partial D$ in general, $d\left(\gamma_{1}, \gamma_{2} ; D\right)$ is the extremal distance between $\gamma_{1}$ and $\gamma_{2}$ in $D$. We note $d([0, i],[M, M+i] ; Q)=$ $M$ and the well known inequality

$$
M_{1}\left(s_{1}, s_{2} ; K\right)+M_{2}\left(s_{1}, s_{2} ; K\right) \leqq d\left(s_{1}, s_{2} ; Q\right) .
$$

Figure 1 gives an example in the case $s_{1}=[0, x], s_{2}=[i, M+i]$.

2. We shall investigate the set of values of the pairs $\left(M_{1}, M_{2}\right)$ obtained this way in its dependence of the chosen pair of sides and the choice of $x$. Here we shall find that certain "extremal" continua which are produced by quadratic differentials play a key role. We shall omit the case that the side $[x, M]$ is selected since a reflection in the line $\{z ; \operatorname{Re} z=M / 2\}$ immediately leads to a situation in which $[0, M-x]$ is a selected side. So we are left essentially with the following three cases:

Case I. $s_{1}=[0, i], s_{2}=[M, M+i], P=x$;

Case II. $s_{1}=[0, x], s_{2}=[M, M+i], P=i$;

Case III. $s_{1}=[0, x], s_{2}=[i, M+i], P=M$.

Of these, we will study first case I giving detailed proofs of the relevant facts; since cases II and III exhibit many similar features, a briefer discussion seems appropriate.

3. Let $M>0$ and $Q=\langle 0, M\rangle \times\langle 0, i\rangle$. In this section, and up to section 8 , we let $s_{1}=[0, i]$ and $s_{2}=[M, M+i]$. We put $S=\langle 0, M\rangle \times$ $\langle 0, M\rangle$. With Weierstrass' $p$-function $p(z ; 2 M, 2 i)=p(z)$ and $(x, y) \in \bar{S}$, we introduce the quadratic differential

$$
\sigma_{x y}=-\frac{1-p(z) / p(y)}{1-p(z) / p(x)} d z^{2}, \quad z \in \bar{Q},
$$

where the formal expression $p(z) / p(0)$ is replaced by 0 for each $z \in \bar{Q}$. Denoting by $K_{x y}$ the closure of the union of the trajectories $\sigma_{x y}>0$ which have the limit point $y$, we describe first the set $K_{x y}$.

(a) $(x, y) \in S^{+}=\{(x, y) ; 0<y<x<M\}$. Then $\sigma_{x y}>0$ has two trajectories with limit point $y$. One trajectory has as its closure the segment $[y, x]$; the other trajectory has as its closure the carrier $K_{x y}^{\prime}$ of an analytic are with initial point $y$ and terminal point on $\langle i, M+i\rangle$ 
which lies up to initial and terminal point in $Q$, forming at $y$ with the direction of the positive real axis the angle $2 \pi / 3$.

(b) $(x, y) \in S^{-}=\{(x, y) ; 0<x<y<M\}$. Then again $\sigma_{x y}>0$ has two trajectories with limit point $y ;[x, y]$ is the closure of one trajectory, the closure $K_{x y}^{\prime}$ of the other trajectory has the same properties as mentioned in (a) except that the angle with the positive real axis at $y$ is now $\pi / 3$.

(c) $(x, y)$ with $0<x=y<M . \sigma_{x y}>0$ has one trajectory with limit point $y$, which is already a closed set, namely $K_{x y}^{\prime}=[y, y+i]$.

In the cases $(\mathrm{a})-(\mathrm{c})$ thus $K_{x y}=[x, y] \cup K_{x y}^{\prime} ;$ considering $Q$ as a pentagon with sides $s_{1}, s_{2}$, and $s_{3}=[0, x], s_{4}=[x, M], s_{5}=$ $[i, i+M]$, we have $K_{x y} \in \mathfrak{K}\left(s_{1}, s_{2} ; x\right)$, and $s_{1}$ as well as $s_{2}$ are trajectories of $\sigma_{x y}>0$.

$\left.\mathrm{d}_{1}\right) x \in\langle 0, M\rangle, y=0$. Then $K_{x 0}=[0, x] \cup s_{1}$, and $s_{2}$ is a trajectory of $\sigma_{x 0}>0$.

$\left.\mathrm{d}_{2}\right) x \in\langle 0, M\rangle, y=M$. Then $K_{x_{M}}=[x, M] \cup s_{2}$, and $s_{1}$ is a trajectory of $\sigma_{x M}>0$.

$\left.\mathrm{e}_{1}\right) \quad x=0, y \in\langle 0, M\rangle$. Then $K_{0 y}=[0, y] \cup K_{0 y}^{\prime}$, where $K_{0 y}^{\prime}$ has the properties of $K_{x y}^{\prime}$ mentioned in b); $s_{2}$ is a trajectory of $\sigma_{0 y}>0$, and $s_{1}$ is the closure of a trajectory.

$\left.\mathrm{e}_{2}\right) x=M, y \in\langle 0, M\rangle$. Then $K_{M y}=[y, M] \cup K_{M y}^{\prime}$, where $K_{M y}^{\prime}$ has the properties of $K_{x y}^{\prime}$ mentioned in a); $s_{1}$ is a trajectory of $\sigma_{M y}>0$ and $s_{2}$ is the closure of a trajectory.

f) $K_{00}=s_{1}$, and $s_{2}$ is a trajectory of $\sigma_{00}>0$;

$K_{M 0}=s_{1} \cup[0, M]$, and $s_{2}$ is the closure of a trajectory of $\sigma_{M 0}>0$

$K_{0 M}=s_{2} \cup[0, M]$, and $s_{1}$ is the closure of a trajectory of $\sigma_{0 M}>0$

$K_{M M}=s_{2}$, and $s_{1}$ is a trajectory of $\sigma_{M M}>0$.

\section{Defining}

$$
\left(\frac{1-p(z) / p(y)}{1-p(z) / p(x)}\right)^{1 / 2}
$$

analytic in $Q$, continuous on $\bar{Q}-\{x\}$, and positive on $[i, M+i]$, we put

$$
f(z)=\int_{y}^{z}\left(-\sigma_{x y}\right)^{1 / 2}, \quad z \in Q .
$$

For $(x, y) \in S^{+} \cup S^{-}, f$ maps $Q$ conformally onto a hexagon bounded by straight line segments parallel to the real or the imaginary axis, with five right angles (corresponding to the points $0, x, M, M+i, i$ ) and 
with one $3 \pi / 2$-angle (corresponding to $y)$; for $(x, y) \in \bar{S}-\left(S^{+} \cup S^{-}\right)$ various degeneracies appear. $\left(\bar{Q}-K_{x y}\right) \cap Q$ either consists of two components (in the cases (a), (b), (c), (e) i.e. when $y \neq 0, M$ ), then we call $D_{j}$ that component $(j=1,2)$ which has $s_{j}$ on the boundary; or $\left(\bar{Q}-K_{x y}\right) \cap Q$ consists of just one component (in the remaining cases), which we call $D_{1}$ when $y=M$, and $D_{2}$ when $y=0$ (they are obtained as limiting cases when $y \rightarrow 0, M)$; for $(x, y) \in S$ we have in the notation of section $1, D_{j}=D_{j}\left(s_{1}, s_{2} ; K_{x y}\right)$.

We put $(j=1,2)$ for $(x, y) \in \bar{S}$

$$
\begin{array}{ll}
M_{j}^{*}(x, y)=d\left(s_{j}, K_{x y} \cap \partial D_{j} ; D_{j}\right) & \text { when } D_{j} \text { is defined, } \\
M_{j}^{*}(x, y)=0 & \text { when } D_{j} \text { is not defined. }
\end{array}
$$

For $(x, y) \in S$ we have thus: $M_{j}^{*}(x, y)=M_{j}\left(s_{1}, s_{2} ; K_{x y}\right)$ is the modulus of the rectangle $f\left(D_{j}\right)$ with respect to its vertical sides. We put further for $(x, y) \in \bar{S}$

$$
\begin{aligned}
& a_{1}(x, y)=\int_{0}^{\min (x, y)}\left|\sigma_{x y}\right|^{1 / 2}, \quad b_{1}(x, y)=\int_{0}^{i}\left|\sigma_{x y}\right|^{1 / 2}, \\
& a_{2}(x, y)=\int_{\max (x, y)}^{M}\left|\sigma_{x y}\right|^{1 / 2}, \quad b_{2}(x, y)=\int_{M}^{M+i}\left|\sigma_{x y}\right|^{1 / 2},
\end{aligned}
$$

the integration being carried out on $[\alpha, \beta]$ when $\alpha$ is the initial and $\beta$ the terminal point in any of the definite integrals (4.3). Simply checking the various cases, we obtain $(j=1,2)$ from (4.3) now

$$
M_{j}^{*}(x, y)=\frac{a_{j}(x, y)}{b_{j}(x, y)}, \quad(x, y) \in \bar{S} ;
$$

indeed (apart from the degenerate cases (d), (e), (f)) the function $f$ maps $D_{j}$ onto a rectangle with horizontal sides of length $a_{j}(x, y)$ and vertical sides of length $b_{j}(x, y)$. We observe further that in (4.3) $a_{1}$ and $a_{2}$ are always finite and non-negative on $\bar{S}$ while $b_{1}$ and $b_{2}$ are positive (possibly infinite) there.

5. While $a_{1}, a_{2}, b_{1}, b_{2}$ are not continuous on $\bar{S}$, we have $\mathrm{L}$ e m m a 1. Let $h: \bar{S} \rightarrow \boldsymbol{R}^{2}$ be defined by

$$
h(x, y)=\left(M_{1}^{*}(x, y), M_{2}^{*}(x, y)\right), \quad(x, y) \in \bar{S} .
$$

Then $h$ is continuous. 
We shall see later (Theorem 2 ) that the restriction of $h$ to $\overline{S^{+}}$or to $\overline{S^{-}}$ is actually a homeomorphism onto the triangle

$$
\bar{\Delta}=\left\{\left(\mu_{1}, \mu_{2}\right) ; 0 \leqq \mu_{1}+\mu_{2} \leqq M, \mu_{1} \geqq 0, \mu_{2} \geqq 0\right\} .
$$

Proof of Lemma 1. The continuity of $h$ on $S \cup(\langle 0, M\rangle \times\{0, M\})$ follows from (4.4) since the functions $a_{1}, b_{1}, a_{2}, b_{2}$ are clearly continuous on this set, by (4.3) and (3.1). To prove the continuity of $h$ on the remaining set $\{0, M\} \times[0, M]$, we observe that by the trajectory structure of the quadratic differentials $\sigma_{x y},(x, y) \in \bar{S}$, the reflection of $K_{x y}$ in the line $\{z ; \operatorname{Re} z=M / 2\}$ yields $K_{M-x, M-y}$, whence

$$
\begin{aligned}
& M_{1}^{*}(M-x, M-y)=M_{2}^{*}(x, y), \quad(x, y) \in \bar{S}, \\
& M_{2}^{*}(M-x, M-y)=M_{1}^{*}(x, y) ; \quad
\end{aligned}
$$

it suffices thus to prove the continuity of $h$ just also on $\{0\} \times[0, M]$.

Consider now the continuity at $(0, \eta), \eta \in[0, M]$. By (4.3), (4.4) we have

$$
M_{1}^{*}(0, \eta)=0, \quad M_{2}^{*}(0, \eta)=\frac{a_{2}(0, \eta)}{b_{2}(0, \eta)} .
$$

The continuity of $M_{2}^{*}$ at $(0, \eta)$ follows since $a_{2}$ and $b_{2}$ are easily seen to be continuous at that point by (4.3), (3.1). The continuity of $M_{1}^{*}$ at $(0, \eta)$ may either be inferred directly (considering the quotient $a_{1}(x, y) / b_{1}(x, y)$ near $(0, \eta)$ which is, however, a little cumbersome when $\eta=0)$ or by using $M_{1}^{*}(x, M) \rightarrow 0$ when $x \rightarrow 0$ together with the inequality $M_{1}^{*}(x, y) \leqq M_{1}^{*}(x, M)$ for each $y \in[0, M]$, which inequality is immediate from the structures of $K_{x y}$ and $K_{x M}$.

6. L e $\mathrm{m} \mathrm{m}$ a 2. Let $x \in\langle 0, M\rangle$ and $K \in \Re\left(s_{1}, s_{2} ; x\right)$. Then for any $y \in[0, M]$ we have the inequality

$$
\begin{gathered}
b_{1}^{2}(x, y) M_{1}\left(s_{1}, s_{2} ; K\right)+b_{2}^{2}(x, y) M_{2}\left(s_{1}, s_{2} ; K\right) \\
\leqq b_{1}^{2}(x, y) M_{1}^{*}(x, y)+b_{2}^{2}(x, y) M_{2}^{*}(x, y) ;
\end{gathered}
$$

for $y=0$ or $M$, the inequality (6.1) is strict ; for $y \in\langle 0, M\rangle$ we have equality in (6.1) if and only if $K=K_{x y}{ }^{1}$

The proof is accomplished in a standard way by the extremal-lengthmethod, using in $Q$ the metric $\left|\sigma_{x y}\right|^{1 / 2}$.

Using the fact that $K_{x y} \in \mathfrak{\pi}\left(s_{1}, s_{2} ; x\right)$ once $(x, y) \in S$, we conclude from (1.1) that $h(S)$ lies in the closed triangle $\bar{\Delta}$ of $(5.2)$, and Lemma 1 yields

\footnotetext{
${ }^{1}$ Note that (6.1) yields (1.1) for $y=x$.
} 


$$
h(\bar{S}) \subset \bar{\Delta}
$$

Writing

$$
\Delta=\left\{\left(\mu_{1}, \mu_{2}\right) ; 0<\mu_{1}+\mu_{2}<M, \mu_{1}>0, \mu_{2}>0\right\}
$$

for the interior of $\bar{\Delta}$ we have

Theorem 1. Let $x \in\langle 0, M\rangle$ and let $h_{x}:[0, M] \rightarrow \boldsymbol{R}^{\mathbf{2}}$ denote the section of $h$ at $x$ given by

$$
h_{x}(y)=\left(M_{1}^{*}(x, y), M_{2}^{*}(x, y)\right), \quad y \in[0, M] .
$$

Then

(i) $h_{x}$ is injective;

(ii) $\Gamma_{x}=h_{x}([0, M])$ is a smooth curve in the closed triangle $\bar{\Delta}$ which has at $h_{x}(y)$ the slope

$$
\lambda_{x}(y)=-b_{1}^{2}(x, y) / b_{2}^{2}(x, y)<0 ;
$$

(iii) the slope $\lambda_{x}$ is strictly decreasing on $[0, M]$ from $\lambda_{x}(0)$ at $h_{x}(0)=$ $\left(0, M_{2}^{*}(x, 0)\right) \in \partial \Delta$ over $\lambda_{x}(x)=-1$ at $h_{x}(x)=(x, M-x) \in \partial \Delta$ to $\lambda_{x}(M)$ at $h_{x}(M)=\left(M_{1}^{*}(x, M), 0\right) \in \partial \Delta$;

(iv) the component $\Delta_{x}$ of $\left(\bar{\Delta}-\Gamma_{x}\right) \cap \Delta$ which has the point $(0,0)$ on the boundary is a convex domain touching the side $[(M, 0),(0, M)]$ of $\Delta$ at just one point, namely $(x, M-x)$.

Proof. To prove (i) we observe $h_{x}(0) \in\langle(0,0),(0, M)\rangle$ and $h_{x}(M) \in\langle(0,0),(M, 0)\rangle$ whence $h_{x}(0) \neq h_{x}(M)$, while for $y_{1} \in\langle 0, M\rangle$ and a different point $y_{2} \in[0, M]$ we obtain $h_{x}\left(y_{1}\right) \neq h_{x}\left(y_{2}\right)$ at once from Lemma 2 choosing there $K=K_{x y_{1}}$ and $y=y_{2}$, since $K_{x y_{1}} \neq K_{x y_{2}}$.

We show now that for fixed $x \in\langle 0, M\rangle$,

$$
\begin{aligned}
& M_{1}^{*}(x, y) \text { is strictly increasing in } y \in[0, M], \\
& M_{2}^{*}(x, y) \text { is strictly decreasing in } y \in[0, M] .
\end{aligned}
$$

First consider $(\partial / \partial y) M_{1}^{*}(x, y)$ for $y \in\langle x, M\rangle$. We have

$$
\begin{aligned}
& \operatorname{sign} \frac{\partial}{\partial y} M_{1}^{*}(x, y) \\
& =\operatorname{sign}\left[b_{1}(x, y) \frac{\partial}{\partial y} a_{1}(x, y)-a_{1}(x, y) \frac{\partial}{\partial y} b_{1}(x, y)\right]
\end{aligned}
$$

and the expression in parentheses in (6.6) equals

$$
\int_{0}^{i}\left|\sigma_{x y}\right|^{1 / 2} \cdot \frac{\partial}{\partial y} \int_{0}^{x}\left|\sigma_{x y}\right|^{1 / 2}-\int_{0}^{x}\left|\sigma_{x y}\right|^{1 / 2} \cdot \frac{\partial}{\partial y} \int_{0}^{i}\left|\sigma_{x y}\right|^{1 / 2}
$$


A little consideration shows that the differentiation can be taken under the integral sign which makes (6.7) equal to

$$
\begin{aligned}
\frac{1}{2} \frac{p^{\prime}(y)}{p^{2}(y)}\left[\int_{0}^{i}\left|\sigma_{x y}\right|^{1 / 2} \cdot \int_{0}^{x} \frac{p(z)}{1-p(z) / p(y)}\left|\sigma_{x y}\right|^{1 / 2}\right. \\
\left.\quad-\int_{0}^{x}\left|\sigma_{x y}\right|^{1 / 2} \cdot \int_{0}^{i} \frac{p(z)}{1-p(z) / p(y)}\left|\sigma_{x y}\right|^{1 / 2}\right] .
\end{aligned}
$$

Since for $t \in\langle 0, x\rangle$ and $\tau \in\langle 0,1]$ we have

$$
\frac{p(t)}{1-p(t) / p(y)}<-p(y)<\frac{p(i \tau)}{1-p(i \tau) / p(y)},
$$

the bracketed expression in (6.8) is negative, and since $p^{\prime}(y)<0,(6.8)$ is positive, whence

$$
\frac{\partial}{\partial y} M_{1}^{*}(x, y)>0 \quad \text { for } y \in\langle x, M\rangle .
$$

Thus $M_{1}^{*}(x, y)$ is strictly increasing for $y \in[x, M]$.

Let now $x \leqq y_{1}<y_{2}<M$. Writing $l_{x y}$ for the right hand side of (6.1), the point $h_{x}\left(y_{1}\right)=\left(M_{1}^{*}\left(x, y_{1}\right), M_{2}^{*}\left(x, y_{1}\right)\right)$, lies trivially on the line

$$
L_{x y_{1}}=\left\{\left(\mu_{1}, \mu_{2}\right) ; b_{1}^{2}\left(x, y_{1}\right) \mu_{1}+b_{2}^{2}\left(x, y_{1}\right) \mu_{2}=l_{x y_{1}}\right\},
$$

which line has the negative slope

$$
\lambda_{x}\left(y_{1}\right)=-b_{1}^{2}\left(x, y_{1}\right) / b_{2}^{2}\left(x, y_{1}\right) .
$$

By Lemma 2, on the other hand, the point $h_{x}\left(y_{2}\right)$ lies strictly below the line (6.10). Since for the first component of these points we have $M_{1}^{*}\left(x, y_{1}\right)<M_{1}^{*}\left(x, y_{2}\right)$, the slope $\lambda_{x}\left(y_{1}, y_{2}\right)$ of $\left[h_{x}\left(y_{1}\right), h_{x}\left(y_{2}\right)\right]$ satisfies

$$
\lambda_{x}\left(y_{1}, y_{2}\right)<\lambda_{x}\left(y_{1}\right), \quad x \leqq y_{1}<y_{2}<M ;
$$

and in particular the second components of $h_{x}\left(y_{1}\right)$ and $h_{x}\left(y_{2}\right)$ fulfill by (6.11) the opposite inequality $M_{2}^{*}\left(x, y_{1}\right)>M_{2}^{*}\left(x, y_{2}\right)$, whence $M_{2}^{*}(x, y)$ is strictly decreasing for $y \in[x, M]$. A similar procedure applied to the interval $[0, x]$ then gives $(6.5)$.

Considering instead of the line (6.10) the line $L_{x y_{2}}$, we obtain instead of $(6.12)$

$$
\lambda_{x}\left(y_{2}\right)<\lambda_{x}\left(y_{1}, y_{2}\right), \quad x \leqq y_{1}<y_{2}<M
$$

both (6.12) and (6.13) are easily verified also when $0<y_{1}<y_{2} \leqq x$, and the continuity of (6.4) for $y \in[0, M]$ finally yields 


$$
\lambda_{x}\left(y_{2}\right)<\lambda_{x}\left(y_{1}, y_{2}\right)<\lambda_{x}\left(y_{1}\right), \quad \text { when } 0 \leqq y_{1}<y_{2} \leqq M .
$$

$\Gamma_{x} \subset \bar{\Delta}$ follows from (6.2) while the remainder of statement (ii) in Theorem 1 is obtained by letting $y_{1}, y_{2} \rightarrow y \in[0, M]$ in formula (6.14) where $y_{1} \leqq y \leqq y_{2}$, and using the continuity of $(6.11)$ in $y_{1} \in[0, M]$.

With (6.4), (6.14) as well as the relation $b_{1}(x, x)=b_{2}(x, x)$, obvious from (3.1) and (4.3), statement (iii) now follows; statement (iv) is easily obtained combining the statements (ii) and (iii).

Remark. Though $\Gamma_{x}$ is a smooth curve with slope -1 at $(x, M-x)$ the partial derivatives of $M_{1}^{*}(x, y), M_{2}^{*}(x, y)$ with respect to $y$ at $y=x$ are infinite.

7. For $(x, y) \in S$, the function $f$ of (4.1) maps $Q$ onto a hexagon $H$ (degenerate to a rectangle when $x=y$ ) which is bounded by straight line segments parallel to the real or the imaginary axis. The imaginary axis decomposes $H=f(Q)$ into two rectangles $H_{1}=f\left(D_{1}\right)$ in the left half plane and $H_{2}=f\left(D_{2}\right)$ in the right half plane. $M_{j}^{*}(x, y),(j=1,2)$, is the extremal distance of the vertical sides of $H_{j}$, while $M$ is the extremal distance of the "outer" vertical sides $f([0, i])$ and $f([M, M+i])$ of $H$ in $H$. The question arises: to what extent does a pair $\left(M_{1}^{*}, M_{2}^{*}\right)$, obtained that way, characterize the pair $(x, y) \in S$. In order to answer that question we need some information about the change of the extremal distance of the outer vertical sides of such a hexagon when one of the rectangles $H_{j}$ is subject to a homothetic transformation.

L e m m a 3 . Let $M>0$ and $\xi \in\langle 0, M\rangle$; let $R=\langle-\xi, M-\xi\rangle \times$ $\langle 0, i\rangle, R_{1}=\langle-\xi, 0\rangle \times\langle 0, i\rangle, R_{2}=\langle 0, M-\xi\rangle \times\langle 0, i\rangle$; for $\left.\vartheta\right\rangle 0$ let $R_{2}(\vartheta)=\langle 0, \vartheta(M-\xi)\rangle \times\langle 0, \vartheta i\rangle$ be the rectangle obtained from $R_{2}$ through homothetic stretching by the factor $\vartheta$, keeping 0 fixed; let

$$
\begin{gathered}
\left.R(\vartheta)=\text { interior of }\left(\bar{R}_{1} \cup \overline{R_{2}(\vartheta}\right)\right)=R(M, \xi, \vartheta), \\
\mu(\vartheta)=d([-\xi,-\xi+i],[\vartheta(M-\xi), \vartheta(M-\xi+i)] ; R(\vartheta)) .
\end{gathered}
$$

Then $^{2} \mu:\langle 0, \infty\rangle \rightarrow \boldsymbol{R}$ is strictly decreasing on $\langle 0,1]$, strictly increasing on $[1, \infty\rangle$, and

$$
\lim _{\vartheta \rightarrow 0} \mu(\vartheta)=\infty=\lim _{\vartheta \rightarrow \infty} \mu(\vartheta) .
$$

We have $R=R(1) ; R(\vartheta)$ is a hexagon of a type similar to that of $H$ above.

Proof of Lemma 3. $\mu(\vartheta) \rightarrow \infty$ for $\vartheta \rightarrow 0$ is obvious. Writing for the moment $\mu(M, \xi, \vartheta)$ instead of $\mu(\vartheta)$ to take into account also the

\footnotetext{
${ }^{2}$ Here the notation $\langle 0, \infty\rangle$ etc. for subsets of the real axis is self-explanatory.
} 
dependence on $M$ and $\xi$, we have $\mu(M, \xi, \vartheta)=\mu(M, M-\xi, 1 / \vartheta)$; $\lim _{\vartheta \rightarrow \infty} \mu(\vartheta)=\infty$ now follows, and we conclude further that the Lemma is proved once it is shown that $\mu$ is just strictly increasing for $\vartheta \in[1, \infty\rangle$. Let now $\vartheta>1$. Abbreviating the vertices of $R(\vartheta)$ by

$$
\begin{aligned}
& A=-\xi+i, \quad B=-\xi, \quad C=\vartheta(M-\xi), \\
& D=\vartheta(M-\xi+i), \quad E=\vartheta i, \quad F=i,
\end{aligned}
$$

we have for the extremal distances $M_{1}, M_{2}$ of the vertical sides of the two rectangles $R_{1}, R_{2}(\vartheta)$ obviously

$$
\begin{aligned}
& M_{1}=d\left([A, B],[0, F] ; R_{1}\right)=\xi, \\
& M_{2}=d\left([0, E],[C, D] ; R_{2}(\vartheta)\right)=M-\xi,
\end{aligned}
$$

whence (observing $\vartheta>1$ ) immediately

$$
\mu(\vartheta)=d([A, B],[C, D] ; R(\vartheta))>M_{1}+M_{2}=M=\mu(1) .
$$

To prove now $\mu\left(\vartheta_{1}\right)>\mu(\vartheta)$ when $1<\vartheta<\vartheta_{1}$, we determine first an extremal metric $\varrho$ for the extremal distance $\mu(\vartheta)$. We select $t_{3}>t_{2}>$ $t_{1}>1$ so that the upper half plane $U$ can be mapped conformally onto $R(\vartheta)$ in such a way that the boundary points $\infty, 0,1, t_{1}, t_{2}, t_{3}$ of $U$ correspond in turn to the vertices $A, B, C, D, E, F$ of $R(\vartheta)$. With suitable $\lambda>0$ the mapping function $\Phi$ is then given by

$$
\Phi(z)=\lambda \int_{0}^{z}\left(\frac{t-t_{3}}{t(t-1)\left(t-t_{1}\right)\left(t-t_{2}\right)}\right)^{1 / 2} d t-\xi, \quad z \in U,
$$

where that branch of the square root is taken which has positive boundary values for $t \in\langle 0,1\rangle$. The function $\varphi$ given by

$$
\varphi(z)=\int_{0}^{z} \frac{d t}{\left[t(t-1)\left(t-t_{1}\right)\right]^{1 / 2}}, \quad z \in U,
$$

again with that branch of the square root which has positive boundary values on $\langle 0,1\rangle$, maps $U$ onto a rectangle $\langle 0, a\rangle \times\langle 0, i b\rangle$ with $a>0, b>0$. Having $w \in R(\vartheta)$ related to $z \in U$ by $w=\Phi(z)$, an extremal metric $\varrho(w)|d w|$ in $R(\vartheta)$ is given by

$$
\frac{|d z|}{\left|z(z-1)\left(z-t_{1}\right)\right|^{1 / 2}}=\frac{1}{\left|z(z-1)\left(z-t_{1}\right)\right|^{1 / 2}} \frac{|d w|}{\left|\Phi^{\prime}(z)\right|},
$$

thus

$$
\varrho(w)=\frac{1}{\lambda}\left|\frac{t_{2}-z}{t_{3}-z}\right|^{1 / 2}, \quad z=\Phi^{-1}(w)
$$


We have then $(w=u+i v)$ for the family $\Gamma$ of curves joining $[A, B]$ and $[C, D]$ in $R(\vartheta)$

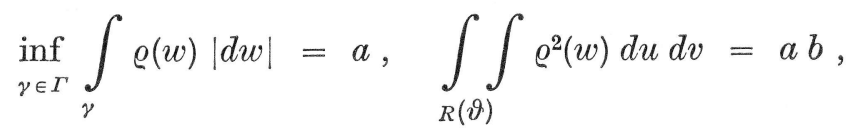

whence

$$
\mu(\vartheta)=\frac{a}{b} .
$$

For small $\varepsilon>0$ we put $\vartheta_{1}=(1+\varepsilon) \vartheta$ and denote the corresponding vertices of $R\left(\vartheta_{1}\right)$ by

$$
\begin{aligned}
& A_{1}=-\xi+i, \quad B_{1}=-\xi, \quad C_{1}=\vartheta_{1}(M-\xi), \\
& D_{1}=\vartheta_{1}(M-\xi+i), \quad E_{1}=\vartheta_{1} i, \quad F_{1}=i .
\end{aligned}
$$

The choice of a suitable metric $\varrho_{1}$ in $R\left(\vartheta_{1}\right)$ will then give $\mu\left(\vartheta_{1}\right)>\mu(\vartheta)$, and the Lemma is proved. ${ }^{3}$

We put $S^{\prime}=\langle 0, C] \times\left[E, E_{1}\right\rangle, S^{\prime \prime}=\left[C, C_{1}\right\rangle \times\left\langle 0, E_{1}\right\rangle$. Noticing that $\varrho$ has a continuous extension to $[E, D] \cup[C, D]$, again denoted by $\varrho$, we put (see Figure 2)

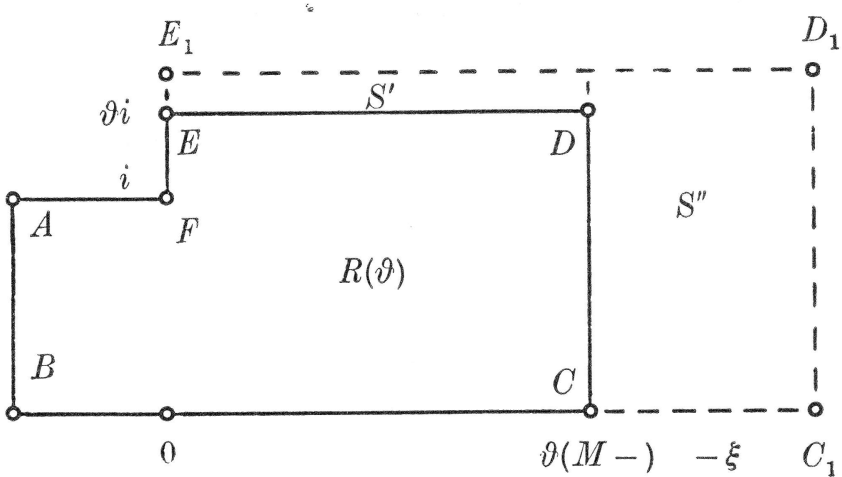

Figure 2

(i) $\varrho_{1}(w)=\varrho(w)$ for $w \in R(\vartheta)$;

(ii) $\varrho_{1}(w)=\varrho(u+i \vartheta)$ for $w=u+i v \in S^{\prime} \quad$ where $u \in\langle 0, C]$ and $v \in\left[\vartheta, \vartheta_{1}\right\rangle$, i.e. $i v \in\left[E, E_{1}\right\rangle$,

(iii) $\varrho_{1}(w)=\varrho(D)$ for $w \in \mathbb{S}^{\prime \prime}$,

observing

${ }^{3}$ A more subtle reasoning, not needed here, gives in fact for the partial derivatives of $d([A, B],[C, D] ; R(\vartheta))$ with respect to each of the variables $\xi, M, \vartheta$ a not too complicated expression. 


$$
\varrho(D)=\frac{1}{\lambda}\left|\frac{t_{2}-t_{1}}{t_{3}-t_{1}}\right|^{1 / 2}=\inf _{w \in[C, D]} \varrho(w) .
$$

If $\Gamma_{1}$ is the family of curves joining $\left[A_{1}, B_{1}\right]=[A, B]$ and $\left[C_{1}, D_{1}\right]$ in $R\left(\vartheta_{1}\right)$, we have

$$
\mu\left(\vartheta_{1}\right) \geqq \frac{L^{2}\left(\Gamma_{1}, \varrho_{1}\right)}{A_{1}\left(\varrho_{1}\right)},
$$

where

$$
L\left(\Gamma_{1}, \varrho_{1}\right)=\inf _{\gamma_{1} \in \Gamma_{1}} \int_{\gamma_{1}} \varrho_{1}(w)|d w|=a+\varrho(D)\left(C_{1}-C\right)
$$

and

$$
\text { (7.14) } \quad A_{1}\left(\varrho_{1}\right)=\iint_{R\left(\vartheta_{1}\right)} \varrho_{1}^{2}(w) d u d v=a b+\int_{S^{\prime} \cup} \int_{S^{\prime \prime}} \varrho_{1}^{2}(w) d u d v \text {. }
$$

Putting $\left(1 \leqq t \leqq t_{2}\right)$

$$
f(t)=\left|t(t-1)\left(t-t_{1}\right)\right|^{-1 / 2}, \quad g(t)=\left|\left(t_{2}-t\right) /\left(t_{3}-t\right)\right|^{1 / 2},
$$

the relations $C_{1}-C=\left(\vartheta_{1}-\vartheta\right)(M-\xi)=\varepsilon \vartheta(M-\xi)=\varepsilon(D-E)$ and (7.11), (7.13), (7.15) yield

$$
L\left(\Gamma_{1}, \varrho_{1}\right)=a+\varepsilon g\left(t_{1}\right) \frac{1}{\lambda}(D-E) .
$$

From (7.6) and (7.15) we obtain further $\left(w \in[E, D], t \in\left[t_{1}, t_{2}\right]\right)$

$$
|d w|=\lambda \frac{f(t)}{g(t)}|d t|, \quad \text { where } w=\Phi(t),
$$

whence with $E_{1}-E=i \vartheta \varepsilon=\varepsilon(D-C)$ and (7.8)

$$
\begin{aligned}
\iint_{S^{\prime}} \varrho_{1}^{2}(w) d u d v & =\left|E_{1}-E\right| \int_{E}^{D} \varrho_{1}^{2}(w)|d w| \\
& =\varepsilon|D-C| \frac{1}{\lambda} \int_{t_{1}}^{t_{2}} g^{2} \frac{f}{g} d t .
\end{aligned}
$$

Formulae (7.11) and (7.15) yield

$$
\begin{aligned}
\int_{S^{\prime \prime}} \int \varrho_{1}^{2} d u d v & =\frac{1}{\lambda^{2}} g^{2}\left(t_{1}\right)\left(C_{1}-C\right)\left|D_{1}-C_{1}\right| \\
& =g^{2}\left(t_{1}\right) \frac{1}{\lambda^{2}} \varepsilon(D-E)(1+\varepsilon)|D-C|
\end{aligned}
$$


Combining (7.14) with (7.16), (7.18), (7.19) we obtain

$$
\begin{aligned}
\mu\left(\vartheta_{1}\right) \geqq & \frac{L^{2}\left(\Gamma_{1}, \varrho_{1}\right)}{A_{1}\left(\varrho_{1}\right)} \\
= & \frac{\left[a+\varepsilon g\left(t_{1}\right) \lambda^{-1}(D-E)\right]^{2}}{a b+\varepsilon \lambda^{-1}|D-C| \int_{t_{1}}^{t_{2}} f g d t+\varepsilon(1+\varepsilon) g^{2}\left(t_{1}\right) \lambda^{-2}(D-E)|D-C|} \\
= & \frac{a}{b} \frac{1+2 \varepsilon g\left(t_{1}\right) \lambda^{-1}(D-E) / a}{1+\frac{\varepsilon}{a b} \lambda^{-1}|D-C| \int_{t_{1}}^{t_{2}} f g d t+\frac{\varepsilon}{a b} g^{2}\left(t_{1}\right) \lambda^{-2}(D-E)|D-C|}+O\left(\varepsilon^{2}\right) \\
= & \frac{a}{b}\left[1+\frac{\varepsilon \lambda^{-1}}{a b}\left\{b g\left(t_{1}\right)(D-E)-|D-C| \int f g d t\right\}\right. \\
& \left.+\frac{\varepsilon \lambda^{-1}}{a b} g\left(t_{1}\right)(D-E)\left\{b-g\left(t_{1}\right) \lambda^{-1}|D-C|\right\}\right]+O\left(\varepsilon^{2}\right) .
\end{aligned}
$$

Using again (7.17) and the fact that $g$ is nonvanishing and strictly decreasing in $t \in\left[1, t_{2}>\right.$ we obtain

$$
\begin{aligned}
(7.21) b g\left(t_{1}\right)(D-E) & =\int_{1}^{t_{1}} f d t \cdot \lambda \int_{t_{1}}^{t_{2}} f(t) \frac{g\left(t_{1}\right)}{g(t)} d t>\int_{1}^{t_{1}} f d t \cdot \lambda \int_{t_{1}}^{t_{2}} f 1 d t \\
& =\lambda \int_{1}^{t_{1}} \frac{f(t)}{g\left(t_{1}\right)} d t \cdot \int_{t_{1}}^{t_{2}} f(t) g\left(t_{1}\right) d t>\lambda \int_{1}^{t_{1}} \frac{f}{g} d t \cdot \int_{t_{1}}^{t_{2}} f g d t \\
& =|D-C| \cdot \int_{t_{1}}^{t_{2}} f g d t,
\end{aligned}
$$

and

$$
\begin{aligned}
b & =\int_{1}^{t_{1}} f d t>\int_{1}^{t_{1}} f(t) \frac{g\left(t_{1}\right)}{g(t)} d t=g\left(t_{1}\right) \cdot \int_{1}^{t} \frac{f}{g} d t \\
& =g\left(t_{1}\right) \lambda^{-1}|D-C| .
\end{aligned}
$$

Using now (7.21) and (7.22) in the last expression of (7.20) and comparing with (7.9) we finally conclude for any sufficiently small $\varepsilon>0$ 


$$
\mu((1+\varepsilon) \vartheta)=\mu\left(\vartheta_{1}\right)>\frac{a}{b}=\mu(\vartheta)
$$

where $\vartheta>1$, thus Lemma 3 is proved.

8. Recalling the notation

$$
\begin{aligned}
& S=\langle 0, M\rangle \times\langle 0, M\rangle, \\
& S^{+}=\{(x, y) ; 0<y<x<M\}, \\
& S^{-}=\{(x, y) ; 0<x<y<M\}, \\
& \Delta=\left\{\left(\mu_{1}, \mu_{2}\right) ; 0<\mu_{1}+\mu_{2}<M, \mu_{1}>0, \mu_{2}>0\right\},
\end{aligned}
$$

we have

Theorem 2. Let $M>0$ and let $h: \bar{S} \rightarrow R^{2}$ be given by (5.1). Then

(i) $h$ maps $\bar{S}$ onto $\bar{\Delta}$;

(ii) the restriction of $h$ to each of the two sets $\bar{S}^{+}$and $\bar{S}^{-}$is a homeomorphism onto $\bar{\Delta}$, this homeomorphism is moreover sense-preserving on $\overline{S^{+}}$ and sense-reversing on $\bar{S}^{-}$.

Proof. We shall prove the part of statement (ii) which concerns $S^{+}$, since in view of (5.3) this suffices to prove the theorem. We shall proceed in several steps.

I. $h: \partial S^{+} \rightarrow \partial \Delta$ is a homeomorphism which is sense-preserving. On $\partial S^{+}=[(0,0),(M, 0)] \cup[(M, 0),(M, M)] \cup[(M, M),(0,0)]$ we have

$$
\begin{aligned}
& h(x, 0)=\left(0, d\left(s_{1} \cup[0, x], s_{2} ; Q\right)\right) \text { for } x \in[0, M], \\
& h(M, y)=\left(a_{1}(M, y) / b_{1}(M, y), 0\right) \text { for } y \in[0, M], \\
& h(x, x)=(x, M-x) \text { for } x \in[0, M] .
\end{aligned}
$$

(8.1) shows that $h$ is injective on $[(0,0),(M, 0)]$ with $h(0,0)=(0, M)$ and $h(M, 0)=[0,0]$, and the continuity of $h$ implies that $h$ maps $[(0,0),(M, 0)]$ homeomorphically onto $[(0, M),(0,0)]$. The reasoning leading to (6.9) likewise shows $(\partial / \partial y)\left(a_{1}(M, y) / b_{1}(M, y)\right)>0$ for $y \in\langle 0, M\rangle$ and one concludes from (8.2) that $h$ maps $[M, 0),(M, M)]$ homeomorphically onto $[(0,0),(M, 0)]$. With (8.3) it is now immediate that $h: \partial S^{+} \rightarrow \partial \Delta$ is a sense-preserving homeomorphism.

II. $h$ is injective on $\bar{S}^{+}$. Since we know already that $h$ is injective on $\partial S^{+}$and since $h(x, y) \in \Delta$ for $(x, y) \in \bar{S}^{+}-\partial S^{+}=S^{+}$we have just to show that $h$ is injective on $S^{+}$.

Let therefore $\left(x_{1}, y_{1}\right),\left(x_{2}, y_{2}\right) \in S^{+}$be such that $h\left(x_{1}, y_{1}\right)=$ $h\left(x_{2}, y_{2}\right)$. Putting $(j=1,2)$ 


$$
f_{j}(z)=\frac{1}{b_{2}\left(x_{j}, y_{j}\right)}\left[\int_{i}^{z}\left(\frac{1-p(t) / p\left(y_{j}\right)}{1-p(t) / p\left(x_{j}\right)}\right)^{1 / 2} d t+a_{1}\left(x_{j}, y_{j}\right)\right], \quad z \in Q,
$$

and choosing that branch of the square root which has negative boundary values on $\left\langle 0, y_{j}\right\rangle$, we have in the notation (7.1)

$$
f_{j}(Q)=R\left(M_{1}^{*}\left(x_{j}, y_{j}\right)+M_{2}^{*}\left(x_{j}, y_{j}\right), M_{2}^{*}\left(x_{j}, y_{j}\right), \vartheta_{j}\right)
$$

where

$$
\vartheta_{j}=b_{1}\left(x_{j}, y_{j}\right) / b_{2}\left(x_{j}, y_{j}\right)<1,
$$

the boundary points $M, M+i, i, 0, y, x$ being mapped in this order to the points $A, B, C, D, E, F$ of (7.4), replacing there $(M, \xi, \vartheta)$ by $\left(M_{1}^{*}\left(x_{j}, y_{j}\right)+M_{2}^{*}\left(x_{j}, y_{j}\right), M_{2}^{*}\left(x_{j}, y_{j}\right), \vartheta_{j}\right)$. From Lemma 3 we conclude $\vartheta_{1}=\vartheta_{2}$, whence $f_{2}^{-1} \circ f_{1}$ is a conformal selfmap of $Q$ keeping the boundary points $0, M, M+i, i$ individually fixed. Thus $f_{2}^{-1} \circ f_{1}$ is the identity $I$ on $Q$, and we obtain

$$
\begin{aligned}
& x_{2}=f_{2}^{-1}\left(f_{1}\left(x_{1}\right)\right)=I\left(x_{1}\right)=x_{1}, \\
& y_{2}=f_{2}^{-1}\left(f_{1}\left(y_{1}\right)\right)=I\left(y_{1}\right)=y_{1},
\end{aligned}
$$

and $\left(x_{1}, y_{1}\right)=\left(x_{2}, y_{2}\right)$, showing that $h$ is indeed injective on $\mathcal{S}^{+}$.

III. $h: \overline{S^{+}} \rightarrow \bar{\Delta}$ is surjective. Since $h: \partial S^{+} \rightarrow \partial \Delta$ was already seen to be surjective, it remains to show that $h: S^{+} \rightarrow \Delta$ is surjective. Let $w \in \Delta$ and assume that $w \notin h\left(S^{+}\right)$. Since $h\left(\partial S^{+}\right) \cap \Delta=\varnothing$ we have also $w \notin h\left(\bar{S}^{+}\right)$. If $\gamma$ is the Jordan curve obtained by positive orientation of the boundary $\partial S^{+}$, the continuity of $h$ on $\bar{S}+$ yields by a standard homotopy argument that the winding number of $h(\gamma)$ with respect to $w$ is zero, which by $h\left(\partial S^{+}\right)=\partial \Delta$ is obviously false. Thus indeed $h\left(\bar{S}^{+}\right)=\bar{\Delta}$.

IV. Since $h$ is a continuous bijection of the compact set $\bar{S}+$ onto $\bar{\Delta}$, $h$ is a homeomorphism, and $h$ is sense preserving since (by step I) it is sense preserving on $\partial S^{+}$, proving the Theorem.

9. Let $(x, y) \in \bar{S} . \quad \sigma_{x y}>0$ determines a directional field in $Q$ prescribing at each point $z \in Q$ the direction

$$
\arg d z=-\frac{1}{2} \arg \left(-\frac{1-p(z) / p(y)}{1-p(z) / p(x)}\right) \bmod \pi .
$$

The direction (9.1) depends apparently continuously on all three variables $(x, y, z) \in[0, M] \times[0, M] \times Q$, and we showed that the pair $h=$ $\left(M_{1}^{*}, M_{2}^{*}\right)$ not only is continuously depending on $(x, y) \in \bar{S}$, but that $h$ is also a homeomorphism when $y$ is restricted to $[0, x]$. 
We want to extend this homeomorphism when the zero $y$ of the quadratic differential $\sigma_{x y}$ is no longer restricted to $[0, x]$ but varies on $\partial Q-\{x\}$. To this end we shall define a directional field in $Q$ also for these values of $y$ in a natural manner. The form (9.1), however, is not suitable since $p\left(y_{0}\right)=0$ for some $y_{0} \in[i, M+i] \cup[M, M+i]$ making (9.1) undefined for $y=y_{0}[(9.1)$ has a limit direction at each point $z \in Q$ for fixed $x$, when $y \rightarrow y_{0}$ on $\partial Q$ from one side; the two limit directions thus obtained differ by $\pi / 2 \bmod \pi$, though].

We put

$$
T=\{(x, y) ; x \in\langle 0, M\rangle, y \in \partial Q-\{x\}\},
$$

and for $(x, y) \in T, z \in \bar{Q}$ we define

$$
\begin{aligned}
\sigma_{x y}^{*} & =-\frac{1+p(x)}{1+|p(y)|} \frac{p(z)-p(y)}{p(z)-p(x)} d z^{2}, \quad \text { when } y \in\langle 0, x\rangle, \\
& =\frac{1+p(x)}{p(z)-p(x)} d z^{2}, \quad \text { when } y=0, \\
& =\frac{1+p(x)}{1+|p(y)|} \frac{p(z)-p(y)}{p(z)-p(x)} d z^{2}, \quad \text { when } y \in \partial Q-[0, x] .
\end{aligned}
$$

For fixed $x \in\langle 0, M\rangle$ the discontinuity of (9.3) in $y$ is now placed at the point $y=x$ since

$$
\lim _{y \rightarrow x-} \sigma_{x y}^{*}=-d z^{2}=\sigma_{x x}, \quad \lim _{y \rightarrow x+} \sigma_{x y}^{*}=+d z^{2}=-\sigma_{x x},
$$

and comparing with (3.1), we have for $(x, y) \in \bar{S}+\cap T$

$$
\sigma_{x y}^{*}=\frac{1+1 / p(x)}{1+1 / p(y)} \sigma_{x y} .
$$

Thus for $(x, y) \in \bar{S}^{+} \cap T, \sigma_{x y}^{*}>0$ and $\sigma_{x y}>0$ have the same trajectories; using $\sigma_{x y}^{*}$ instead of $\sigma_{x y}$ in (4.3) and (4.4), the quantities (4.3) pick up the factor $(1+1 / p(x)) /(1+1 / p(y))>0$ while the quotient (4.4) remains unchanged. Moreover, the directional field defined in $Q$ by $\sigma_{x y}^{*}>0$ apparently depends continuously on $((x, y), z) \in T \times Q \cdot[T$ is topologized in the natural manner, carrying over the product topology of $\langle 0, M\rangle \times \partial Q$.]

For $(x, y) \in T$ we denote by $K_{x y}^{*}$ the closure of the union of the trajectories of $\sigma_{x y}^{*}>0$ which have the limit point $y$. Thus first

$$
K_{x y}^{*}=K_{x y} \quad \text { for } \quad(x, y) \in \bar{S}^{+} \cap T=T_{0} .
$$

Letting 


$$
\begin{aligned}
& T_{1}=\langle 0, M\rangle \times(\langle 0, i] \cup[i, M+i\rangle), \\
& T_{2}=(\langle 0, M\rangle \times[M, M+i\rangle) \cup S^{-},
\end{aligned}
$$

and observing $T=T_{0} \cup T_{1} \cup(\langle 0, M\rangle \times\{M+i\}) \cup T_{2}$ we obtain in the other cases

$$
\begin{gathered}
K_{x y}^{*}=[y, i] \cup K_{x y}^{\prime} \quad \text { for }(x, y) \in T_{1}, \\
K_{x, M+i}^{*}=[i, M+i] \cup[M, M+i] \quad \text { for } x \in\langle 0, M\rangle, \\
K_{x y}^{*}=[y, M] \cup K_{x y}^{\prime} \quad \text { for }(x, y) \in T_{2} .
\end{gathered}
$$

Here $K_{x y}^{\prime}$ is the carrier of an analytic arc with initial point $y$ and terminal point (in case $(9 b))$ on $\langle x, M\rangle$ or (in case $(9 d)$ ) on $\langle 0, i\rangle$, the arc lying up to initial and terminal point in $Q .[M, M+i]$ is a trajectory of $\sigma_{x y}^{*}>0$ for $(x, y) \in T_{1}$, while $[i, i+M]$ is a trajectory when $(x, y) \in T_{2}$; $[0, x]$ is the closure of a trajectory in all three cases $(9 \mathrm{~b}),(9 \mathrm{c}),(9 \mathrm{~d})$. Considering $Q$ as a pentagon as in section 1, we have

$$
\begin{array}{ll}
K_{x y}^{*} \in \Re([0, x],[M, M+i] ; i) & \text { for }(x, y) \in T_{1}, \\
K_{x y}^{*} \in \Re([0, x],[i, M+i] ; M) & \text { for }(x, y) \in T_{2},
\end{array}
$$

leading to the cases II and III of section 2. We put therefore

$$
\begin{aligned}
s_{1}(x, y) & =[0, x] \quad \text { for }(x, y) \in T_{1} \cup T_{2}, \\
s_{2}(x, y) & =[M, M+i] \quad \text { for }(x, y) \in T_{1}, \\
& =[i, M+i] \quad \text { for }(x, y) \in T_{2} .
\end{aligned}
$$

10. We define now on $T$ a function $h^{*}$, coinciding with $h$ of (5.1) on $S^{+}$, in a natural way, obtaining results which are analogous to the Lemmata 1, 2, 3 and the Theorems 1, 2.

Observing (9.6) and (9.7) we put in the notation of section 1, using (9.8) and (9.9),

$$
\begin{array}{ll}
D_{1}^{*}(x, y)=D_{1}\left(s_{1}(x, y), s_{2}(x, y) ; K_{x y}^{*}\right), & (x, y) \in T_{1} \cup T_{2}, \\
D_{2}^{*}(x, y)=D_{2}\left(s_{1}(x, y), s_{2}(x, y) ; K_{x y}^{*}\right), & (x, y) \in T_{1} \cup T_{2} .
\end{array}
$$

Again, $(j=1,2), K_{x y}^{*} \cap \partial D_{j}^{*}$ is connected for $(x, y) \in T_{1} \cup T_{\mathbf{2}}$, so we put

$$
\begin{array}{r}
M_{1}^{*}(x, y)=-d\left(s_{1}(x, y), K_{x y}^{*} \cap \partial D_{1}^{*}(x, y) ; D_{1}^{*}(x, y)\right) \\
\text { for }(x, y) \in T_{1} \cup T_{2}, \\
M_{1}^{*}(x, M+i)=-d\left([0, x],[i, M+i] \quad \begin{array}{r}
\cup \\
\text { for } x \in\langle 0, M+i] ; Q),
\end{array}\right.
\end{array}
$$




$$
\begin{array}{r}
M_{2}^{*}(x, y)=d\left(s_{2}(x, y), K_{x y}^{*} \cap \partial D_{2}^{*}(x, y) ; \begin{array}{l}
\left.D_{2}^{*}(x, y)\right) \\
\text { for }(x, y) \in T_{1},
\end{array}\right. \\
\begin{aligned}
M_{2}^{*}(x, M+i)= & \text { for } x \in\langle 0, M\rangle, \\
= & -d\left(s_{2}(x, y), K_{x y}^{*} \cap \partial D_{2}^{*}(x, y) ; D_{2}^{*}(x, y)\right) \\
& \text { for }(x, y) \in T_{2},
\end{aligned}
\end{array}
$$

and define $h^{*}$ on $T$ using (10.2), (10.3) and the notation of (9a):

$$
h^{*}(x, y)= \begin{cases}h(x, y) & \text { for }(x, y) \in T_{0} \\ \left(M_{1}^{*}(x, y), M_{2}^{*}(x, y)\right) & \text { for }(x, y) \in T-T_{0} .\end{cases}
$$

The quantities (10.2), (10.3) have expressions similar to (4.4): We have

$$
\begin{aligned}
& M_{1}^{*}(x, y)= \begin{cases}\frac{a_{1}^{*}(x, y)}{b_{1}^{*}(x, y)}, & (x, y) \in T-(\langle 0, M\rangle \times\{0\}), \\
0, & (x, y) \in\langle 0, M\rangle \times\{0\},\end{cases} \\
& M_{2}^{*}(x, y)= \begin{cases}\frac{a_{2}^{*}(x, y)}{b_{2}^{*}(x, y)}, & (x, y) \in T-(\langle 0, M\rangle \times\{M+i\}), \\
0, & (x, y) \in\langle 0, M\rangle \times\{M+i\},\end{cases}
\end{aligned}
$$

where $a_{1}^{*}, b_{1}^{*}, a_{2}^{*}, b_{2}^{*}$ are obtained from the table below in the

Table 1

\begin{tabular}{c|cccc}
$y$ & $a_{1}^{*}$ & $b_{1}^{*}$ & $a_{2}^{*}$ & $b_{2}^{*}$ \\
\hline$[x, 0]$ & & & & \\
0 & {$[0, y]$} & {$[0, i]$} & {$[x, M]$} & {$[M, M+i]$} \\
{$[0, i]$} & 0 & - & {$[x, M]$} & {$[M, M+i]$} \\
{$[i, M+i]$} & $-[0, y]$ & {$[0, x]$} & {$[i, M+i]$} & {$[M, M+i]$} \\
$M+i$ & $-[0, i]$ & {$[0, x]$} & {$[y, M+i]$} & {$[M, M+i]$} \\
{$[M+i, M]$} & $-[0, i]$ & {$[0, x]$} & 0 & - \\
{$[M, x]$} & $-[x, M]$ & {$[0, x]$} & $-[y, M+i]$ & {$[i, M+i]$} \\
& $-[x, y]$ & {$[0, x]$} & $-[M, M+i]$ & {$[i, M+i]$}
\end{tabular}

following way: if $x \in\langle 0, M\rangle$ and $y \in\langle\alpha, \beta\rangle$ when $[\alpha, \beta]$ is an entry in the first column, if $c \in\left\{a_{1}^{*}, b_{1}^{*}, a_{2}^{*}, b_{2}^{*}\right\}$, and if $[\gamma, \delta]$ or $-[\gamma, \delta]$ is the entry determined by $[\alpha, \beta]$ and $c$, then (10.6) $c(x, y)=\int_{\alpha}^{\beta}\left|\sigma_{x y}^{*}\right|^{1 / 2} \quad$ or $\quad-\int_{\alpha}^{\beta}\left|\sigma_{x y}^{*}\right|^{1 / 2}$, integration on $[\alpha, \beta]$; 
the expression (10.6) is used also for $y \in\{\alpha, \beta\}-\{x\}$ excepting $c=b_{1}^{*}, y=0$ and $c=b_{2}^{*}, y=M+i$, leaving (as indicated by dashes in Table 1) $b_{1}^{*}$ undefined at $(x, 0)$ and $b_{2}^{*}$ undefined at $(x, M+i)$; in each case, however, $\lim _{y \rightarrow \alpha} c(x, y)$ and $\lim _{y \rightarrow \beta} c(x, y)$ do exist and are finite, and we put in particular

(10.7) $\lim _{\substack{y \rightarrow 0 \\ y>0}} b_{1}^{*}(x, y)=b_{1}^{*}(x, 0+)>0, \lim _{\substack{y \rightarrow 0 \\ i y<0}} b_{1}^{*}(x, y)=b_{1}^{*}(x, 0-)>0$,

$$
\begin{aligned}
\lim _{\substack{y \rightarrow M \\
y<M}} b_{2}^{*}(x, i+y) & =b_{2}^{*}(x,(M+i)+)>0, \\
\lim _{\substack{y \rightarrow 1 \\
y<1}} b_{2}^{*}(x, M+i y) & =b_{2}^{*}(x,(M+i)-)>0 .
\end{aligned}
$$

Though the intervals in the first column of Table 1 overlap, an application of Cauchy's Integral Theorem shows at once: $a_{1}^{*}$ and $a_{2}^{*}$ are well defined on $T, b_{1}^{*}$ is well defined on $T-(\langle 0, M\rangle \times\{0\}), b_{2}^{*}$ is well defined on $T-(\langle 0, M\rangle \times\{M+i\})$. We have e.g.

$$
a_{1}^{*}(x, y)=-\int_{0}^{y}\left|\sigma_{x y}^{*}\right|^{1 / 2} \quad \text { for }(x, y) \in\langle 0, M\rangle \times[0, i] .
$$

Now we may write instead of (10.4)

$$
h^{*}(x, y)=\left(M_{1}^{*}(x, y), M_{2}^{*}(x, y)\right) \quad \text { for }(x, y) \in T .
$$

L e m m a 4 . a) The functions $a_{1}^{*}, b_{1}^{*}, a_{2}^{*}, b_{2}^{*}$ are continuous on their respective domains of definition, $b_{1}^{*}$ and $b_{2}^{*}$ are positive, and we have for each $x \in\langle 0, M\rangle$

$$
\begin{aligned}
& b_{1}^{*}(x, 0+)+b_{1}^{*}(x, 0-)=b_{2}^{*}(x, 0), \\
& b_{2}^{*}(x,(M+i)+)+b_{2}^{*}(x,(M+i)-)=b_{1}^{*}(x, M+i) ;
\end{aligned}
$$

b) the functions $M_{1}^{*}, M_{2}^{*}$ are continuous on $T$.

The easy proof, similar to parts of the proof of Lemma 1, is omitted.

$\mathrm{L} \mathrm{e} \mathrm{m} \mathrm{m} \mathrm{a} 5$. Let $x \in\langle 0, M\rangle$, let $s_{1}=[0, x], s_{2}=[M, M+i]$ and $K \in \mathfrak{K}\left(s_{1}, s_{2} ; i\right)$. Then for any $y \in\langle 0, i] \cup[i, M+i\rangle$ we have the inequality

$$
\begin{aligned}
& b_{1}^{* 2}(x, y) M_{1}\left(s_{1}, s_{2} ; K\right)+b_{2}^{* 2}(x, y) M_{2}\left(s_{1}, s_{2} ; K\right) \\
& \leqq b_{1}^{* 2}(x, y)\left(-M_{1}^{*}(x, y)\right)+b_{2}^{* 2}(x, y) M_{2}^{*}(x, y),
\end{aligned}
$$

with equality if and only if $K=K_{x y}^{*}$; for $y \in\{0, M+i\}$ formula (10.11) holds with strict inequality when $b_{1}^{*}(x, y)$ and $b_{2}^{*}(x, y)$ are replaced by the corresponding limits. 
L e m m a 6 . Let $x \in\langle 0, M\rangle$, let $s_{1}=[0, x], s_{2}=[i, M+i]$ and $K \in \mathfrak{I}\left(s_{1}, s_{2} ; M\right)$. Then for any $y \in\langle M+i, M] \cup[M, x\rangle$ we have the inequality

$$
\begin{aligned}
& b_{1}^{* 2}(x, y) M_{1}\left(s_{1}, s_{2} ; K\right)+b_{2}^{* 2}(x, y) M_{2}\left(s_{1}, s_{2} ; K\right) \\
\leqq & b_{1}^{* 2}(x, y)\left(-M_{1}^{*}(x, y)\right)+b_{2}^{* 2}(x, y)\left(-M_{2}^{*}(x, y)\right),
\end{aligned}
$$

with equality if and only if $K=K_{x y}$; for $y=M+i$, formula (10.12) holds with strict inequality when $b_{2}^{*}(x, y)$ is replaced by $b_{2}^{*}(x,(M+i)-)$; we have also

$$
x^{2} M_{1}\left(s_{1}, s_{2} ; K\right)+M^{2} M_{2}\left(s_{1}, s_{2} ; K\right)<M .
$$

The proofs of Lemma 5 and Lemma 6 are analogous to the proof of Lemma 2; (10.13) is obtained from (10.12) using

$$
\begin{aligned}
& \lim _{\substack{y \rightarrow x \\
y>x}} b_{1}^{*}(x, y)=x, \quad \lim _{\substack{y \rightarrow x \\
y>x}} b_{2}^{*}(x, y)=M, \\
& \lim _{\substack{y \rightarrow x \\
y>x}} M_{1}^{*}(x, y)=0, \quad \lim _{\substack{y \rightarrow x \\
y>x}} M_{2}^{*}(x, y)=-\frac{1}{M},
\end{aligned}
$$

where these latter relations follow easily from (9.3), (10.5), Table 1.

11. For $x \in\langle M, 0\rangle$ we use now the two-point compactification $\partial \hat{Q}_{x}$ of $Q-\{x\}$ by adding the two points

$$
x-=\lim _{\substack{y \rightarrow x \\ y<x}} y \text { and } x+=\lim _{\substack{y \rightarrow x \\ y>x}} y .
$$

We put further

$$
\begin{gathered}
\Delta_{1}=\left\{\left(\mu_{1}, \mu_{2}\right) ; \mu_{1}<0,0<\mu_{2}<M\right\}, \\
\Delta_{2}=\left\{\left(\mu_{1}, \mu_{2}\right) ; \mu_{1}<0,-1 / M<\mu_{2}<0\right\},
\end{gathered}
$$

and with $\bar{\Delta}$ of $(5.2)$,

$$
\begin{aligned}
& \bar{E}=\bar{\Delta} \cup \bar{\Delta}_{1} \cup \bar{\Delta}_{2}, \\
& E=\text { interior of } \bar{E} .
\end{aligned}
$$

Then we have in analogy to Theorem 2

Th e orem 3 . Let $x \in\langle 0, M\rangle$ and let

$$
\partial \hat{Q}_{x}=(\partial Q-\{x\}) \cup(\{x-\} \cup\{x+\})
$$

be the two-point compactification of $\partial Q-\{x\} ;$ let $h_{x}^{*}: \partial \hat{Q}_{x} \rightarrow R^{2}$ be the continuous function, satisfying 


$$
h_{x}^{*}(y)=\left(M_{1}^{*}(x, y), M_{2}^{*}(x, y)\right) \quad \text { for } y \in \partial Q-\{x\} .
$$

Then

(i) $h_{x}^{*}$ is injective;

(ii) $\Gamma_{x}^{*}=h_{x}^{*}\left(\partial \hat{Q}_{x}\right)$ is a piecewise smooth curve in $\bar{E}$, the points $h_{x}^{*}(0)$ and $h_{x}^{*}(M+i)$ being the points at which the slope of $\Gamma_{x}^{*}$ is discontinuous ;

(iii) $h_{x}^{*}(\partial Q-\{x\}) \subset E$, in particular

$$
\begin{gathered}
h_{x}^{*}(\langle 0, x\rangle) \subset \Delta, \\
h_{x}^{*}(\langle 0, i] \cup[i, M+i\rangle) \subset \Delta_{1}, \\
h_{x}^{*}(\langle M+i, M] \cup[M, x\rangle) \subset \Delta_{2},
\end{gathered}
$$

and $h_{x}\left(\partial \hat{Q}_{x}\right)$ is a cross-cut of $E$;

(iv) for $y \in\langle x, 0\rangle \cup\langle 0, i] \cup[i, M+i\rangle \cup\langle M+i, M] \cup[M, x\rangle, \Gamma_{x}^{*}$ has at $h_{x}^{*}(y)$ the slope

(11.5) $\quad \lambda_{x}^{*}(y)$

$$
\begin{aligned}
& =-b_{1}^{* 2}(x, y) / b_{2}^{* 2}(x, y)<0, \quad y \in\langle x, 0\rangle \cup\langle M+i, M] \cup[M, x\rangle, \\
& =b_{1}^{* 2}(x, y) / b_{2}^{* 2}(x, y)>0, \quad y \in\langle 0, i] \cup[i, M+i\rangle ;
\end{aligned}
$$

(v) the angle $\varphi_{x}^{*}(y) \in\langle 0,2 \pi\rangle$ which the tangent-vector to $\Gamma_{x}^{*}$, oriented in the sense corresponding to the negative orientation of $\partial Q$, forms at $h_{x}^{*}(y)$ with the direction of the positive $\mu_{1}$-axis in the $\left(\mu_{1}, \mu_{2}\right)$-plane is strictly increasing from

$$
\varphi_{x}^{*}(x-)=3 \pi / 4 \quad \text { at } h_{x}^{*}(x-)=(x, M-x)
$$

to

$$
\varphi_{x}^{*}(x+)=\arctan \lambda_{x}^{*}(x+) \in\langle 7 \pi / 4,2 \pi\rangle \quad \text { at } h_{x}^{*}(x+)=(0,-1 / M),
$$

over

$$
\begin{aligned}
& \varphi_{x}^{*}(0+)=\arctan \lambda_{x}^{*}(0+) \in\langle 3 \pi / 4, \pi\rangle, \\
& \varphi_{x}^{*}(0-)=\arctan \lambda_{x}^{*}(0-) \in\langle\pi, 5 \pi / 4\rangle, \\
& \varphi_{x}^{*}(i)=5 \pi / 4, \\
& \varphi_{x}^{*}((M+i)+)=\arctan \lambda_{x}^{*}((M+i)+) \in\langle 5 \pi / 4,3 \pi / 2\rangle, \\
& \varphi_{x}^{*}((M+i)-)=\arctan \lambda_{x}^{*}((M+i)-) \in\langle 3 \pi / 2,7 \pi / 4\rangle, \\
& \varphi_{x}^{*}(M)=7 \pi / 4 ;
\end{aligned}
$$

(vi) the map $x \rightarrow \varphi_{x}^{*}(x+)$ is a decreasing homeomorphism of $\langle 0, M\rangle$ onto $\langle 7 \pi / 4,2 \pi\rangle$.

Proof. That $h_{x}^{*}$ is continuous on $\partial Q-\{x\}$ with continuous extension to $\partial \hat{Q_{x}}$ follows from $(10.5)$ with table 1 , and one obtains in particular 
$h_{x}^{*}(x+)=(0,-1 / M)$. The restriction of the statements of Theorem 3 to $\langle 0, x-\rangle \in \partial \hat{Q}_{x}$ is contained in Theorem 1. The proof of Theorem 1 was based on Lemma 2 and the expression (4.4). Using now Lemma 5 for $y \in\langle 0, i] \cup[i, M+i\rangle$ and Lemma 6 for $y \in\langle M+i, M] \cup[M, x\rangle$ together with the expressions (10.5), a similar reasoning gives those statements of Theorem 3 which concern $y \in\langle 0, i] \cup[i, M+i\rangle \cup\langle M+i, M] \cup$ $[M, x\rangle$. With $h_{x}^{*}(0) \in\left(\bar{\Delta} \cup \bar{\Delta}_{1}\right) \cap E$ and $h_{x}^{*}(M+i) \in\left(\bar{\Delta}_{1} \cup \overline{\Delta_{2}}\right) \cap E$ the statements (i) through (v) of Theorem 3 follow. Finally, (10.14) and (11.5) give immediately statement (vi), completing the proof.

12. We recall the definition (9.2) of $T$ and the topology of $T$. Denoting for $(x, y) \in T$ by $d_{x y} \in\langle 0,2+2 M\rangle$ the distance of $y$ from $x-$ along $O Q$, the map

$$
(x, y) \rightarrow\left(x, x-d_{x y}\right), \quad(x, y) \in T,
$$

is a homeomorphism of $T$ onto the parallelogram

$$
\tilde{T}=\{(x, y) ; 0<x<M,-2-2 M+x<y<x\} ;
$$

the restriction of this homeomorphism to $S^{+} \subset T$ is the identity, and we orient $T$ by carrying over the usual positive orientation of the plane set $\tilde{T}$. Likewise we compactify $T$ by carrying over the compactification of $\tilde{T}$; we call $\hat{T}$ this compactification of $T$ (which corresponds to the compactification $\partial \hat{Q}_{x}$ of $\left.Q-\{x\}\right)$. In the notation (10.9), (11.4) we have as an extension of Theorem 2:

Theorem 4. The map $h^{*}: T \rightarrow E$ is a sense-preserving homeomorphism.

To prove Theorem 4, we adapt the proof of Theorem 2, minor changes are due to the fact that $h^{*}$ cannot be extended homeomorphically to $\hat{T}$ (already $h^{*}(x, x+)=(0,-1 / M)$ for each $\left.x \in\langle 0, M\rangle\right)$. We proceed again in several steps.

I. $h^{*}(T) \subset E$ follows from Theorem 3 (iii).

II. $h^{*}$ is injective on $T$. We shall indicate later how this can be seen.

III. $h^{*}: T \rightarrow E$ is surjective. We use the fact (Theorem 3 ) that for $x \in\langle 0, M\rangle, \Gamma_{x}^{*}$ is a cross-cut of $E$ joining the boundary points $(x, M-x)$ and $(0,-1 / M) \cdot \Gamma_{x}^{*}$ decomposes $E$ into two simple connected components, we denote by $E_{x}$ that component which has the point $(0,0)$ on the boundary. Using II we conclude further: if $\xi \in\langle x, M\rangle$ then $\Gamma_{\xi}^{*}$ is a cross-cut in $E_{x}$. So in order to prove that $h^{*}$ takes the value $\mu=\left(\mu_{1}, \mu_{2}\right) \in E$, it suffices, by a standard homotopy-argument, to show that there exist $x \in\langle 0, M\rangle$ and $\xi \in\langle x, M\rangle$ such that $\mu \in E_{x}$ and that $\Gamma_{\xi}^{*}$ separates 
$\mu$ from $(0,0)$ in $E_{x}$, since, as is easily seen, $h^{*}$ has a continuous extension to the closure $\bar{T}_{x \xi}$ of

$$
T_{x \xi}=\{(t, y) ; t \in\langle x, \xi\rangle, y \in \partial Q-\{t\}\}
$$

in $\hat{T}$.

Assume first $\mu=\left(\mu_{1}, \mu_{2}\right) \in E$ satisfies $\mu_{1}<0, \mu_{2} \geqq 0$. From Theorem 3 it follows that $\mu \in E_{x}$ once there exists $y \in\langle 0, i] \cup[i, M+i]$ such that $M_{1}^{*}(x, y)<\mu_{1}, M_{2}^{*}(x, y)>\mu_{2}$. To find such a pair $(x, y)$ we choose first $y \in\langle 0, i]$ such that

$$
\mu_{2}<\int_{i}^{M+i}\left|\frac{1-p(z) / p(y)}{1+1 /|p(y)|}\right|^{1 / 2}|d z| / \int_{M}^{M+i}\left|\frac{1-p(z) / p(y)}{1+1 /|p(y)|}\right|^{1 / 2}|d z| .
$$

This is possible since the quotient in (12.2) tends to $M$ for $y \rightarrow 0$, $y \in\langle 0, i]$, and since $\mu_{2}<M$ for $\mu \in E$. From (9.3) we have then for $x \in\langle 0, M\rangle$

$$
\sigma_{x y}^{*}=\frac{1+1 / p(x)}{1+1 /|p(y)|} \frac{1-p(z) / p(y)}{p(z) / p(x)-1} d z^{2},
$$

and comparison with (12.2) yields: there exists $x_{0} \in\langle 0, M\rangle$ such that $M_{2}^{*}(x, y)>\mu_{2}$ once $x \in\left\langle 0, x_{0}\right\rangle$, since $\left|\sigma_{x y}^{*}\right|^{1 / 2}$ tends uniformly to the integrands in (12.2) for $x \rightarrow 0$. Using the Laurent-series of $p(z)$ near $z=0$ it is easily seen that

$$
b_{1}^{*}(x, y)=\int_{0}^{x}\left|\sigma_{x y}\right|^{1 / 2}
$$

is bounded for fixed $y$ and $x \in\left\langle 0, x_{0}\right\rangle$, while

$$
a_{1}^{*}(x, y)=-\int_{0}^{y}\left|\sigma_{x y}^{*}\right|^{1 / 2}
$$

tends to $-\infty$ when $x \rightarrow 0, x>0$. Thus for suitable $x \in\left\langle 0, x_{0}\right\rangle$ we have $M_{1}^{*}(x, y)<\mu_{1}$ and $M_{2}^{*}(x, y)>\mu_{2}$ as desired, and $\mu \in E_{x}$. If now $\xi \in\langle x, M\rangle$ is such that $\mu \notin \bar{E}_{\xi}$ then $\Gamma_{\xi}^{*}$ separates $\mu$ from $(0,0)$ in $E_{x}$. By Theorem 3 again $\mu \notin \bar{E}_{\xi}$ certainly holds when $M_{1}^{*}(\xi, M+i)>\mu_{1}$ since $M_{2}^{*}(\xi, M+i)=0$, and (observing $\mu_{1}<0$ ) this is by (10.2) obviously the case when $\xi$ is sufficiently close to $M$. Similar considerations in the other cases $\left(\mu_{1} \geqq 0\right.$ or equivalently $\mu \in \bar{\Delta} \cap E$ already covered in the proof of Theorem 2, and $\mu_{2}<0$ or equivalently $\mu \in \Delta_{2}$ ) show again the existence of $x \in\langle 0, M\rangle$ and $\xi \in\langle x, M\rangle$ so that $\mu \in E_{x}$ 
and that $\Gamma_{\xi}^{*}$ separates $\mu$ from $(0,0)$ in $E_{x}$. So any value $\mu \in E$ is indeed taken by $h^{*}$ in $T$.

IV. $h^{*}: T \rightarrow E$ is a homeomorphism. To see that it suffices now to show that $h^{*}$ is an open mapping. We show therefore: if $\left(x_{0}, y_{0}\right)$ is any point of $T$ and if $G$ is a sufficiently small open set containing $\left(x_{0}, y_{0}\right)$ then $h^{*}(G)$ is open. We choose $x, \xi$ so that $0<x<x_{0}<\xi<M$ and that $\Gamma_{\xi}^{*}$ separates $\mu=h^{*}\left(x_{0}, y_{0}\right)$ from $(0,0)$ in $E_{x}$. The cross-cut $\Gamma_{\xi}^{*}$ decomposes $E_{x}$ into two components, we call $E_{x \xi}$ that component which has $\Gamma_{x}^{*}$ on the boundary, and we note

$$
\partial E_{x \xi}=[(\xi, M-\xi),(x, M-x)] \cup \Gamma_{x}^{*} \cup \Gamma_{\xi}^{*} .
$$

We have $\mu \in E_{x \xi}$, and the continuous extension of $h^{*}$ to $\bar{T}_{x \xi}$ satisfies $h^{*}(t, t-)=(t, M-t)$ and $h^{*}(t, t+)=(0,-1 / M)$, for $t \in[x, \xi]$. The homotopy-argument of III then implies

$$
E_{x \xi}=h^{*}\left(T_{x \xi}\right) .
$$

Since $h^{*}(G) \subset E_{x \xi}$ once $G$ is sufficiently small, by (12.3), we have

$$
h^{*}(G)=E_{x \xi} \cap\left[R^{2}-h^{*}\left(\bar{T}_{x \xi}-G\right)\right]
$$

and since $h^{*}\left(\bar{T}_{x \xi}-G\right)$ is compact, $h^{*}(G)$ is by (12.4) clearly open. So Theorem 4 is proved once it is shown that $h^{*}: T \rightarrow E$ is injective, which fact will be dealt with now.

13. In section 8 , the proof that the function $h$ is injective on $\bar{S}^{+}$, did use Lemma 3. By similar reasoning it is shown that $h^{*}: T_{1} \rightarrow \Delta_{1}$ is injective by the use of

$\mathrm{L}$ e m m a 7. Let $M, \xi, \vartheta, R(\vartheta)$ be as in Lemma 3; let

$$
\begin{aligned}
\mu_{1}(\vartheta) & =d([-\xi+i, i],[\vartheta(M-\xi), \vartheta(M-\xi+i)] ; R(\vartheta)) \quad \text { for } 0<\vartheta \leqq 1, \\
& =d([-\xi+i, i] \cup[i, i \vartheta],[\vartheta(M-\xi), \vartheta(M-\xi+i)] ; R(\vartheta)) \\
& \text { for } 1<\vartheta<\infty .
\end{aligned}
$$

Then $\mu_{1}$ is strictly decreasing for $\vartheta \in\langle 0, \infty\rangle$.

Concerning the proof of Lemma 7 , the following remarks may suffice. For $\vartheta \in\langle 0,1]$, the Lemma can be proved by the method used in the proof of Lemma 3 ; the same method can be used to show that the conjugate extremal distance

$$
\frac{1}{\mu_{1}(\vartheta)}=d([-\xi,-\xi+i] \cup[-\xi, \vartheta(M-\xi)],[\vartheta i, \vartheta(M-\xi+i)] ; R(\vartheta))
$$

is strictly increasing for $\vartheta \in[1, \infty\rangle$. 
In a similar way it is proved that $h^{*}: T_{2} \rightarrow \Delta_{2}$ is injective. Since in the notation of section $9, \quad T=T_{0} \cup T_{1} \cup(\langle 0, M\rangle \times\{M+i\}) \cup T_{2}$, since $h^{*}$ was already seen to be injective on $T_{0}$ (Theorem 2), since the sets $h^{*}\left(T_{0}\right), h^{*}\left(T_{1}\right), h^{*}(\langle 0, M\rangle \times\{M+i\}), h^{*}\left(T_{2}\right)$ all are disjoint, and since by $(10.2) h^{*}$ is clearly injective on $\langle 0, M\rangle \times\{M+i\}$, $h^{*}: T \rightarrow E$ is indeed injective, completing the proof of Theorem 4 .

Technische Universität Berlin

Fachbereich Mathematik

D 1 Berlin 12

Received 3 September 1975 